\title{
Modelling risks to manufacturer and buyer for lifetime warranty policies
}

\author{
Anisur Rahman ${ }^{1 *}$, Gopinath Chattopadhyay ${ }^{2}$ \\ 1 Affiliation Griffith School of Engineering, Griffith University, Gold Coast, Qld 9726, Australia \\ 2 Affiliation Central Queensland University, Gladstone campus, Qld 4680, Australia
}

(Received 13 February 2008, Revised 18 August 2009, Accepted 20 January 2010)

\begin{abstract}
Currently, manufacturers/dealers have started selling products with lifetime warranty policies. In this paper, risk attitudes of both buyers and manufacturer/dealers to lifetime warranty policies are discussed. In line with Chun and Tang (1995) [2], Risk models are developed for products with time dependent failure intensity (rate). These models have been proposed with Non homogeneous Poisson's process for failure intensity function, a constant repair cost, and concave utility function. Using the exponential utility function, the decision models are developed to maximise the manufacturer/dealer's certainty profit equivalent. Risk preference models are developed to find the optimal warranty price through the use of the manufacturer's utility function for profit and the buyer's utility function for repair costs. Finally, the sensitivity of the warranty price models is analysed using numerical examples.
\end{abstract}

Keywords: lifetime warranty, risk preferences, warranty price, buyer's repair cost

\section{Introduction}

Large numbers of products have been coming to market and being sold with long-term warranty policies in the form of extended warranties, warranty for used products, service contracts or lifetime warranty policies. Lifetime warranties are becoming popular as they provide assurance to buyer for long reliable service and a better peace of mind. Although the lifetime warranty market has grown over the past five years, there is little published research in this area.

Under the situation of a lifetime warranty transaction, a buyer of a product pays for the warranty at the time of product purchase which in some occasion is factored into the product price. The manufacturer/dealer provides rectification service in case of product failures due to design, manufacturing and quality assurance problems during the defined life of the product. Under such situation, both the manufacturer and the buyer are exposed to uncertainties and risks of warranty pricing and product performance during the lifetime of the product.

By offering a lifetime warranty for a product, the manufacturer is risking in warranty pricing that whether its offer for such warranty will be accepted by the buyers. At the same time, buyers are unsure about the benefits of buying products sold with lifetime warranty policies. Anticipation of higher product failures encourages a buyer to pay for higher warranty price which in turn encourages the manufacturer to charge a higher warranty price. In case of product covered with warranty, the buyer returns to the original manufacturer for rectification of product failures. But when the product is not covered by warranty, the buyer may take it to other service providers for rectifications. If buyers pay higher repair price for any product failures, they might be interested to get warranty cover and even ready to pay a higher warranty price for free repair or replacements. This may encourages the manufacturer to lower the warranty price to attract more buyers and to compete effectively in the market.

Therefore, there is a need to model optimal warranty price which includes both buyers and manufacturers risk preferences. Limited work so far takes into account the manufacturer and buyer's risk preferences toward how much a manufacturer should charge and how much a buyer is willing to pay for a lifetime warranty service. Ritchken and Tapiero (1986) [7] proposed a framework in which warranty policies for non-repairable items can be evaluated according to risk preferences of both the manufacturer/dealer and the buyers where they emphasized the design and pricing of warranties to which the manufacturers are indifferent in an expected utility sense. Given the price warranty schedule, a buyer's response is expressed by selecting the pricewarranty which minimizes disutility. As result a manufacturer can increase profit by tailoring price-warranty schedules to specify buyer's need. Menezes (1989) [4] developed a conceptual framework to examine the impact of warranties on consumer's preferences. Based on the framework, predictions of consumer preferences between product with and without a warranty were derived for various consumer segments.

Chun and Tang (1995) [2] proposed a warranty model for the free-replacement, fixed-period warranty policy that determines the optimal warranty price for a given warranty period. They assumed a constant failure rate for the product, constant repair costs throughout the warranty period, and a producer's and customers' risk aversion for future repair costs. Using the exponential utility function and the gamma failure rate distribution, they derived the decision

\footnotetext{
* Correspondence to: E-mail address: a.rahman@griffith.edu.au.
} 
model that maximizes the producer's certainty profit equivalent. Zhou et al. (2009) [9] proposed a dynamic pricing and warranty policies for products with fixed lifetime where they considered a repairable product with known market entry and departure times and heterogeneous customer attitudes in their risk toward uncertain repair at the expiry of the warranty.

In line with Chun and Tang (1995) [2], this paper proposes the warranty price models that maximize the manufacturer/dealers certainty equivalent by applying Nonhomogeneous Poisson process (NHPP) for products with time dependent failure mode. The reasons behind choosing the product with time depended failure mode are: firstly, most real life products fail due to aging i.e. failure rate increases as the products becomes older and secondly, because of high competition and public demand, quality control system of most consumer durable product through the application of Total quality management, Six Sigma etc has improved so much that the concept of Bath-tub type failure is now almost invalid due very insignificant infant failure.

The outline of this paper is as follows. Section 1 briefly introduces manufacturers' and buyers' risk preferences towards a lifetime warranty policy. Section 2 provides brief overview of lifetime warranty policies. In Section 3, risk preference models for both manufacturer and buyer towards a lifetime warranty policy are developed using exponential utility function. Sensitivity analyses of these models are carried out in Section 4 to see the effect of various factors on the warranty price from both buyer's and manufacturer's point of view. Finally, in the concluding section contribution of this research work and some recommendation of future research scopes are discussed.

\section{Lifetime warranty policies}

Because of market demand for longer life capital intensive assets and huge competition, the manufacturers are under pressure to extend the coverage period or to provide longer term after-sale service through warranty and service contracts. Rahman and Chattopadhyay (2006) [6] developed a framework for long-term warranty policies (see Fig. $1)$. Detail discussions can be found in Rahman and Chattopadhyay (2006) [6]. This paper only looks into the lifetime warranty policies. Lifetime warranty means the manufacturer/dealer's commitment to provide free or cost sharing repair or replacement/s of the sold product in case of failure due to design, manufacturing defects or quality problems throughout the useful life of the product or the buyer's ownership period of the product.

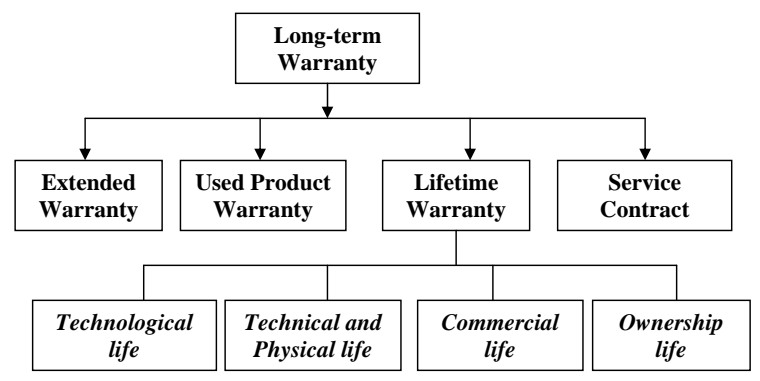

Fig. 1 A framework for the long-term warranty measure

In case of lifetime warranty, it is often difficult to tell about life measures for the period of coverage. The lifetime of the product is assumed to be terminated in some finite, random time horizon. Termination of such warranty may arise from any one of the following reasons (Chattopadhyay and Rahman, 2007 [1]).

Technical life/Physical life: the period over which the product might be expected to last physically, or when replacement or major rehabilitation is physically required.

Technological life: the period until technological obsolescence dictating replacement due to the development of a technologically superior alternative.

Commercial life/Economic life/Functional life: the period, over which the need for the product exists, the period until economic obsolescence dictates replacement with a lower cost alternative (economic or operational convenience).

Ownership life/Social and legal life: the period until human desire or legal requirement dictates replacement or change of ownership occurs.

Although the concept of lifetime warranty has attracted significant attention among the practitioners, publications on lifetime warranty are very limited. Wells (1985) [8] proposed a computational expression for the total expected discounted future cost for a lifetime warranty policy. Chattopadhyay and Rahman (2007) [1] proposed warranty cost models associated with offering lifetime warranty. This paper (Chattopadhyay and Rahman, 2007 [1]) proposed taxonomy for lifetime warranty policies and mathematical models for predicting failures and estimating costs for different lifetime warranty policies. They explored into the potentials of these policies for application in the industry.

\section{Model formulation}

\subsection{Notations and statistical preliminaries}

Let the number of items sold by the manufacturer, be $S$ and $p$ be the proportion of product sold with lifetime warranty and $1-p$ be the proportion of $\mathrm{S}$ products sold without such warranty. Two conditions are considered here: products are sold with warranty or without warranty. Subscripts $m$ and $b$ stands for manufacturer and buyer respectively.

$k \quad:$ The proportion of buyers without warranty coming back to manufacturer for repairing of the faults/defects and $1-k$ portion of buyers prefer to go to third parties for repairing the failed products;

$N_{b}(L) \quad$ : The number of valid claims made by the buyer per item and $N(L)$ is the total number of possible claims for $S$ products over the lifetime $L$;

$E\left[N_{b}(L)\right]$ : The expected number of failure per item experienced by the buyers over the lifetime;

$U_{m}(Y) \quad$ : The manufacturers continuous utility functions for a manufacturer's profit $Y$;

$u_{b}(X) \quad$ : The buyers continuous utility functions for a yer's repair cost $X$;

$U_{m} \quad:$ An individual manufacturer's utility function;

$U_{b} \quad$ : The aggregate utility function representing the entire buyer's risk preference as a whole;

$\Lambda_{m}(t) \quad$ : The manufacturer's failure intensity function;

$\Lambda_{b}(t) \quad$ : The per item failure intensity function for an individual buyer during the lifetime;

$r_{b} \quad$ : The cost of rectification (repair cost) for a buyer in each occasion of failure if the item is not warranted.

When all rectifications are carried out by the manufacturer for some technical reasons, the policy is called manufacturer's technical monopoly. This paper assumes there is no manufacturer's technical monopoly; 
$r_{m}$ : The manufacture's per occasion cost of rectifification (repair cost) which is the actual rectification cost when the item is covered under warranty;

$W$ : The warranty price offered by the manufacturer during the time of purchase.

Product cumulative failure distribution $F(t)$ with density function $f(t)$ in general (assumption), where, $f(t)=$ $\mathrm{d} F(t) / \mathrm{d} t$ and the failure intensity function can be expressed by

$$
\Lambda(t)=\frac{f(t)}{1-F(t)} .
$$

\subsection{Modeling risks in lifetime warranty}

\section{Assumptions}

- Item failures are statistically independent.

- Item failure, in a probabilistic sense, is only a function of its age.

- The time to carry out a rectification action by repair or replacement is negligible compared to the mean time between failures and this time can be ignored.

- An item failure results in an immediate claim and all claims are valid.

- Failures over the warranty period modeled at the system (or item) level.

- Manufacturer's cost of rectification $\mathrm{rm}$ and the buyer's cost of each rectification $r_{b}$ are constant over the warranty period.

- The Manufacturer's utility function $U_{m}(Y)$ for profit $Y$ is concave (risk averter) and strictly increasing as most manufacturers prefer a higher profit to a lower profit. Thus, it follows from Jensen's inequality (Zlobec, 2004 [10]; Keeney and Raiffa, 1976 [3]), that if $Y$ has finite mean then

and

$$
E\left[U_{m}(Y)\right]<U_{m}[E(Y)]
$$

$$
\left[y_{1}<y_{2}\right] \leftrightarrow\left[U_{m}\left(Y_{1}\right)<U_{m}\left(Y_{2}\right)\right]
$$

- The buyer's utility function $U_{b}(X)$ for repair cost $X$ is concave (also risk averter) and strictly decreasing as buyer prefer a lower cost to a higher cost. Thus, it follows from Jensen's inequality that if $X$ has finite mean then

and

$$
E\left[U_{b}(X)\right]<U_{b}[E(X)]
$$

$$
x_{1}<x_{2} \leftrightarrow\left[U_{b}\left(X_{1}\right)>U_{b}\left(X_{2}\right)\right]
$$

Buyer's acceptances of warranty

In determining the worthy of buying such warranty, a buyer may first estimate the total repair cost $r_{b} N_{b}(L)$ of his purchased product during the defined lifetime and then compare it with the given warranty price $W$ in terms of the expected utility. Since $r_{b}$ is constant, buyer's total repair cost is given by $r_{b} N_{b}(L)$ which is estimated by his perceived product failure intensity $\Lambda_{b}(t)$. The higher the buyer estimate of the product failure rate, the more likely he would be willing to buy the warranty.

Let $n_{b}^{*}$ be the number of product failure when buyers are indifferent between the warranty price $W$ and the total repair cost $r_{b} N_{b}(L)$ in terms of the expected utility. Certainty equivalent of similar concept can be found in Moskowitz and Plante (1984) [5]. By definition, a certainty equivalent of $W$ is an amount of $r_{b} N_{b}(L)$ such that the decision maker is indifferent between $W$ and $r_{b} N_{b}(L)$, therefore, it is given by

$$
\begin{aligned}
U_{b}(W) & =E\left[U_{b}\left(r_{b} N_{b}(L)\right)\right] \\
E\left[U_{b}\left(r_{b} N_{b}(L)\right)\right] & =\sum_{n_{b}=0}^{\infty} U_{b}\left(r_{b} n_{b}\right) P\left(N_{b}(L)=n_{b}^{*}\right) .
\end{aligned}
$$

Therefore, we can write

$$
U_{b}(W)=E\left[U_{b}\left(r_{b} N_{b}(L)\right)\right]=\sum_{n_{b}=0}^{\infty} U_{b}\left(r_{b} n_{b}\right) P\left(N_{b}(L)=n_{b}^{*}\right) .
$$

But when failures of product follows Non-homogeneous Poisson process that is failures are time dependent then we have the following equation

$$
\operatorname{Prob}\left(N_{b}(L)=n_{b}^{*}\right)=\frac{\left(\int_{0}^{L} \Lambda_{b}(t) \mathrm{d} t\right)^{n_{b}^{*}} e^{-\int_{0}^{L} \Lambda_{b}(t) \mathrm{d} t}}{n_{b}^{*} !} .
$$

One form for $\Lambda_{b}(t)$ using Non-homogeneous Poisson process

is as follows:

$$
\left[F(t)=1-\exp \left(-\left({ }_{b} \mathrm{t}\right)^{\mathrm{fi}_{\mathrm{b}}}\right)\right]
$$

$$
\Lambda_{b}(t)=\lambda_{b} \beta_{b}(t)^{\left(\beta_{b}-1\right)}
$$

with the shape parameters $\beta_{b}>1$ and inverse characteristic life $\lambda_{b}>0$. This is an increasing function of time or age $t$. Let the buyers' risk aversions be represented by exponential utility functions,

$$
U_{b}(Y)=-e^{c Y}, c>0
$$

Where $c$ is the risk parameters representing the buyers' risk preferences. A buyer's attitude toward risk can be determined by the nature of $c$. A buyer is more risk-averse if the risk parameter increases. A risk parameter $c=0$, indicates a risk neutral buyer. In this study, the buyers' risk parameter $c$ represents a wide spectrum of buyers' risk preferences.

In the exponential utility functions, the absolute risk aversion measure is constant for all $X$, implying that the exponential function represents only the constant risk-averse case (Chun and Tang, 1995 [2]), [10].

Therefore, from Eq. (3) and Eq. (6) we can express the Eq. (7)

$$
e^{c W}=\sum_{n_{b}^{*}=0}^{\infty} e^{c r_{b} n_{b}^{*}} P\left(N_{b}(L)=n_{b}^{*}\right) .
$$

By combining Eq. (4), Eq. (5) and Eq. (7), we can express the Eq. (8)

$$
e^{c W}=\sum_{n_{b}^{*}=0}^{\infty} e^{c r_{b} n_{b}^{*}} \frac{\left(\int_{0}^{L} \lambda_{b} \beta_{b}(t)^{\left(\beta_{b}-1\right)} \mathrm{d} t^{n_{b}^{*}} e^{\left.-\int_{0}^{L} \lambda_{b} \beta_{b}(t)^{\left(\beta_{b}-1\right) \mathrm{d} t}\right)}\right.}{n_{b}^{*} !} .
$$

This can be expressed as Eq. (9)

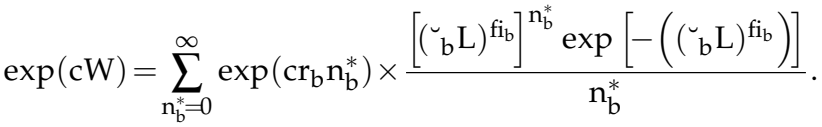

By taking logarithm on both sides of the Eq. (9) we get the buyer's expected warranty price as in Eq. (10) 


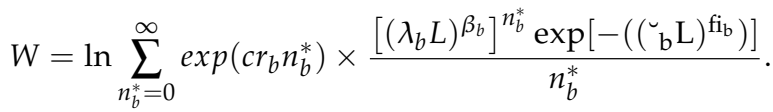

From the above equation it will be worthy for the buyer to accept the warranty offer if the buyer's estimated number of failure is more than and equal to indifferent failure $n_{b}^{*}$. Accordingly, a buyer whose expected failure is higher than $n_{b}^{*}$ would buy the warranty if the total estimated repair cost is higher than the warranty price $W$. Notice that probability to buy this warranty is determined by $n_{b}^{*}$, which, in turn, is determined by $W$.

Based on the information about the buyer's willingness to pay for the warranty price $W$, a manufacturer may determine the warranty price such that the expected total profits during the warranty period is maximized.

Manufacturer's profit The manufacturer's expected total profit $E[\pi(L)]$ for warranty and rectification related services during the lifetime under such warranty policy can be expressed as

$$
\begin{aligned}
E[\pi(L)]= & p\left(S W-E[N(L)] r_{m}\right)+(1-p) k\left[E[N(L)] r_{b}\right. \\
& \left.-E[N(L)] r_{m}\right] \\
= & p\left(S W-E[N(L)] r_{m}\right)+(1-p) k E[N(L)]\left(r_{b}-r_{m}\right) .
\end{aligned}
$$

For a risk-averse manufacturer with an increasing utility function $U_{m}(Y)$, the certainty equivalent for profit can be obtained from the following relationship.

$$
\begin{aligned}
U_{m}(E[\pi(L)])= & U_{m}\left(p\left(S W-E[N(L)] r_{m}\right)\right. \\
& \left.+(1-p) k E[N(L)]\left(r_{b}-r_{m}\right)\right) .
\end{aligned}
$$

As the manufacturer estimated failure intensity is time dependent, it follows the Non-homogeneous Poisson process (NHPP) and here the intensity function can be expressed as

$$
\Lambda_{m}(t)=\lambda_{m} \beta_{m}(t)^{\left(\beta_{m}-1\right)}
$$

with the shape parameters $\beta_{m}>1$ and inverse characteristic life parameter $\lambda_{m}>0$. This is an increasing function of $t$.

Since the items are statistically similar then with more information on product failure manufacture can have probability of $n_{m}$ failures over life $\mathrm{L}$ for any item as given by:

$$
\begin{aligned}
\operatorname{Prob}\left(\mathrm{N}_{\mathrm{m}}(\mathrm{L})\right. & \left.=n_{m}\right)=\frac{\left\{\int_{0}^{L} \Lambda_{m}(t) \mathrm{d} t\right\}^{n_{b}^{*}} e^{-\int_{0}^{L} \Lambda_{m}(t) \mathrm{d} t}}{n_{m} !} ; \\
E\left[N_{m}(L)\right] & =\lambda_{m}^{\beta_{m}}\left(L^{\beta_{m}}\right) ; \\
E[N(L)] & =S \lambda_{m}^{\beta_{m}}\left(L^{\beta_{m}}\right) .
\end{aligned}
$$

Where $a$ is the risk parameters representing the manufacturer's risk preferences. $a>0$ indicates risk averse manufacturer, whereas $a<0$ indicates risk seeker and $a=0$ means a risk neutral manufacturer.

Manufacturers profits for non warranted product, is given by Eq. (11) when $p=0$. Utility of expected profit for warranty and no-warranty strategy for indifferent manufacturer's decision point is as follows:

$$
\begin{aligned}
& e^{-a\left[p S\left(W-\left(\lambda_{m} L\right)^{\beta_{m}} r_{m}\right)+(1-p) S k\left(\lambda_{m} L\right)^{\beta_{m}}\left(r_{b}-r_{m}\right)\right]} \\
= & \sum_{n_{m}=0}^{\infty} e^{-a\left(r_{b}-r_{m}\right) S k n_{m}} \frac{\left[\int_{0}^{L} \lambda_{m} \beta_{m} t^{\left(\beta_{m}-1\right)}\right]^{n_{m}} e^{-\int_{0}^{L} \lambda_{m} \beta_{m} t^{\left(\beta_{m}-1\right)}}}{n_{m}} .
\end{aligned}
$$

Let the difference between the buyer's repair cost at each occasion of failure and the manufacturer's actual cost of repair or rectification $\left(r_{b}-r_{m}\right)$ be $d$ and the proportion of non-warranted buyers $(1-p)$ be $q$.

Eq. (17) can be rewritten as Eq. (18) when it follows the Non-homogeneous Poisson process and taking inverse logarithm on both the sides of the Eq. (17)

$$
\begin{aligned}
& -a\left[p S\left(W-\left(\lambda_{m} L\right)^{\beta_{m}} r_{m}\right)+q S k\left(\lambda_{m} L\right)^{\beta_{m}} d\right] \\
& =\ln \sum_{n_{m}=0}^{\infty} e^{-a d S k n_{m}} \frac{\left[\left(\lambda_{m} L\right)^{\beta_{m}}\right] e^{-\left(\lambda_{m} L\right)^{\beta_{m}}}}{n_{m} !}
\end{aligned}
$$

Optimal warranty price can be derived from Eq. (18) as

$$
\begin{aligned}
W= & -\frac{1}{a p S} \times \ln \sum_{n_{m}=0}^{\infty} e^{-a d S k n_{m}} \frac{\left[\left(\lambda_{m} L\right)^{\beta_{m}}\right] e^{-\left(\lambda_{m} L\right)^{\beta_{m}}}}{n_{m} !} \\
& +\frac{q}{p} k\left(\lambda_{m} L\right)^{\beta_{m}}\left(r_{b}-r_{m}\right)-\left(\lambda_{m} L\right)^{\beta_{m}} r_{m} .
\end{aligned}
$$

\section{Sensitivity analysis of the risk models}

Models developed in this paper appear analytically intractable. Search algorithm and simulation can be used to explore the various parameters and warranty price combinations attractive to buyer and the manufacture's point of view. This is influenced by the perception of the buyers on failure intensity during warranty period, buyers and manufacturer's risk preference and costs associated with repair/rectifications.

In line with Chun and Tang (1995) [2], a sensitivity analysis of the buyer's intension to pay for warranty price is presented with respect to the following factors: (1) buyer's risk preference, (2) buyer's anticipated product failure intensity, and (3) buyer's repair costs, if not warranted. Secondly, we present analysis of the effect of manufacturer offered of warranty price on (1) manufacturer's risk preference, (2) manufacturer anticipated product failure intensity, and (3) buyer's repair cost return rate to the original manufacturer for repair if not warranted.

\subsection{Sensitivity analysis of buyer's willingness to pay for warranty price}

For all cases it is assumed that $60 \%$ of the sold products are with warranty and $40 \%$ products are sold without this type of warranty, which implies that $p=0.6$ and $q=0.4$ for all occasions this analysis.

(1) Effect of buyer's risk preferences on the warranty price

In this numerical analysis part, the buyer's risk parameters ' $c$ ' is varied systematically from 0.1 to 1.0 representing a wide spectrum of buyer's risk preferences. A buyer become more risk averse as the risk parameter ' $c$ ' increases and he/she becomes risk neutral if the parameter is zero.

For the purpose of simplicity, in this sensitivity analysis it is assumed that the buyer's anticipated lifetime of the product $L=3$ years, the buyer's anticipated nonhomogeneous Poisson process parameters: inverse characteristic life $\lambda=0.325$ /year, and shape parameter $\beta=2$, buyer's repair cost of each failure $r_{b}=\$ 30$, if not warranted and the buyer's repair cost is same for all occasions whether it is repaired by the manufacturer/dealer or by an outside repairer. A computer simulation program generates the Tab. 1 for the buyer's acceptance of warranty price with variation of the buyer's risk preference parameter and the buyer's number of failure over the lifetime. 
Table 1 Warranty price $(W)$ In $\$$ for different buyer's risk preferences $(C)$

\begin{tabular}{|c|c|c|c|c|c|}
\hline & \multicolumn{5}{|c|}{$\mathrm{c}$} \\
\hline $\begin{array}{l}\text { Buyer's risk } \\
\text { parameter } c\end{array}$ & 1 & 2 & 3 & 4 & 5 \\
\hline 0.1 & 20.5 & 43.6 & 62.67 & 78.88 & 92.9 \\
\hline 0.3 & 26.66 & 54.18 & 80.35 & 105.56 & 130.03 \\
\hline 0.5 & 27.99 & 56.51 & 84.21 & 111.34 & 138.01741 \\
\hline 0.7 & 28.56 & 57.51 & 85.87 & 113.81 & 141.44 \\
\hline 1 & 28.99 & 58.25 & 87.1 & 115.67 & 144.01 \\
\hline
\end{tabular}

Tab. 1 clearly states the increasing trend of warranty price with the increase of the buyer's risk preference which indicates that the warranty price increases as the buyer becomes more averse and the warranty price decreases as the buyer becomes less averse. This means that the buyer with higher risk averseness is willing to pay higher warranty price. Manufacturer or dealers can use this buyer's psychology while being pricing the warranty. Table 1 also shows that the buyer's willingness to pay warranty price increases as the number of failure increases. This shows that the buyers are ready to pay higher warranty price as their anticipated number of product failure increases.

(2) Effect of buyer's repair cost on the warranty price

Although the buyer's repair cost is applicable only for the buyers with non-warranted item, it has a significant effect on the warranty price. In this analysis, the buyer's rectification or repair cost for each failure is varied systematically from $\$ 30$ to $\$ 70$ representing a wide range of buyer's repair cost. Note that it is assumed this repair costs are constant for a particular product all along its lifetime and the variation is made for policy purpose. Other variables are kept as above and the buyer's risk parameter $c=0.5$. The computer program generates the Tab. 2 for the buyer's acceptance of warranty price with variation of the buyer's repair cost. This shows that the buyer's willingness for warranty price increases linearly as the buyer's repair cost increases. This implies that the buyers are ready to pay higher warranty price if the repair costs are higher. That is, higher proportion of buyers will be interested to buy warranty as the repair price goes up. On the contrary, the buyer's intent to pay less warranty price if its repair costs are less.

Table 2 Warranty price $(W)$ In $\$$ for different buyer's repair $\operatorname{cost}\left(R_{b}\right)$

\begin{tabular}{r|rrrrr}
\hline & \multicolumn{5}{|c}{$n_{b}$} \\
\hline$r_{b}$ & \multicolumn{1}{|c}{1} & \multicolumn{1}{c}{2} & \multicolumn{1}{c}{3} & \multicolumn{1}{c}{4} & \multicolumn{1}{c}{5} \\
\hline 30 & 27.99 & 56.51 & 84.21 & 111.34 & 138.0174 \\
40 & 37.99 & 76.5 & 114.21 & 151.33 & 188.02 \\
50 & 47.99 & 96.6 & 144.23 & 191.34 & 238 \\
60 & 58.01 & 116.51 & 174.22 & 231.33 & 288.02 \\
70 & 67 & 136.51 & 204 & 271.3 & 338 \\
\hline
\end{tabular}

(3) Effect of buyer's anticipated product failure intensity on the warranty price

Here, the sensitivity of buyer's anticipated product failure rate or intensity of product failure on the buyer's acceptance of warranty price is analyzed. To examine the influence of product failure rate on the warranty price, $\lambda_{b}$ is varied from 0.125 to 0.525 per year keeping all other parameters as above. The computer simulation program gen- erates the Tab. 3 for the buyer's accepted warranty prices with variation of the failure intensity parameter $\lambda_{b}$.

Table 3 Effect of buyer's anticipated failure rate on the warranty price

\begin{tabular}{c|ccccc}
\hline & \multicolumn{5}{|c}{$n_{b}$} \\
\hline$\lambda$ & 1 & 2 & 3 & 4 & 5 \\
\hline 0.125 & 25.80 & 50.49 & 74.37 & 97.67 & 120.53 \\
0.225 & 27.52 & 54.18 & 80.35 & 106.44 & 131.65 \\
0.325 & 28.00 & 56.51 & 80.79 & 111.34 & 138.02 \\
0.425 & 28.56 & 57.81 & 86.08 & 114.28 & 142.03 \\
0.525 & 28.99 & 58.63 & 86.91 & 115.95 & 144.55 \\
\hline
\end{tabular}

Tab. 3 shows that the buyer's willingness for warranty price increases as the buyer's anticipated failure intensity increases. This implies that the buyers are ready to pay higher warranty price for higher intensity of product failure. Conversely, when the buyers anticipate a lower intensity of product failure, they will be willing to pay less warranty price.

\subsection{Sensitivity analysis of the manufacture's warranty price}

In this subsection, the sensitivity of manufacturer's optimal warranty pricing is analyzed with the variation of manufacturer's risk preference, manufacturer estimated product failure intensity, buyer's repair cost and buyer return rate to the manufacturer/dealer for repair of the failed product respectively.

(1) Effect of manufacturer/dealer's risk preference on the warranty price

Similar to the sub-section B, claws (1), the manufacturer/dealer risk parameters ' $a$ ' is varied systematically from 0.05 to 1.0 representing a wide spectrum of manufacturer/dealer risk preferences. A manufacturer/dealer become more risk averse as the risk parameter ' $a$ ' increases and the manufacturer/dealer is said to be risk neutral if its risk parameter ' $a$ ' is zero.

Let the expected lifetime of the product $L$ be 3 years, the manufacturer/dealer's estimated non-homogeneous Poisson process parameters are: inverse characteristic life $\lambda_{m}=$ $0.325 /$ year, and shape parameter $\beta_{m}=2$ (this indicates an increasing rate of failure over time), buyer's repair cost for each failure $r_{b}=\$ 30$ and manufacturer's actual cost of each repair $r_{b}=\$ 10$, so that the difference between these two repair costs $d$ is $\$ 20$. It is also assumed that the rate of returning of buyers to the manufacturer/dealer for repair of failed product is $20 \%$ (that is $k=0.2$ ). A computer program generates Tab. 4 exhibiting the influence of buyer's risk preference parameter and the buyer's number of failure over the expected lifetime of the product on manufacturer/dealer's optimal warranty price.

Table 4 Warranty price $(W)$ In $\$$ for different manufacturer's risk preferences $A$ and $N$

\begin{tabular}{l|rrrcc}
\hline & \multicolumn{5}{|c}{$n_{m}$} \\
\hline \multicolumn{1}{c|}{$\mid$} & \multicolumn{1}{|c}{1} & \multicolumn{1}{c}{2} & \multicolumn{1}{c}{3} & 4 & 5 \\
\hline 0.05 & 25.426 & 30.224 & 32 & 32.8 & 33.07 \\
0.1 & 29.93 & 32.08 & 32.9 & 33.14 & 33.22 \\
0.4 & 33.3 & 33.6 & 33.7 & 33.71 & 33.75 \\
0.8 & 34 & 34.1024 & 34.106 & 34.12 & 34.15 \\
1 & 34.23 & 34.25 & 34.257 & 34.26 & 34.33 \\
\hline
\end{tabular}


It is observed that the warranty price increases with the increase of the of the manufacture/dealers risk preference parameter $a$. The warranty price increases as the manufacturer/dealer becomes more risk averse and the warranty price decreases as the manufacturer/dealer becomes less averse. This implies that the more a manufacturer is risk averse the more he/she will charge for the warranty.

It is also observed that the manufacturer's charge for warranty increases as the number of failure increases. This means that the manufacturer/dealer must charge higher warranty price for higher number of product failures to avoid a loss.

(2) Effect of manufacturer/dealer's estimated product failure intensity on the warranty price

In this analysis, it considers a range of manufacturer/dealer's estimated inverse characteristic life $\lambda_{m}$ of product to represent failure intensity (rate) with a view to observe the effect on the manufacturer's optimal charge for warranty. To do so, $\lambda_{m}$ is varied from a range of $0.125 /$ year to $0.443 /$ year. Here, the manufacturer/dealer risk parameters ' $a$ ' is kept constant as 0.4 . Keeping all other variables as above, a computer program generates the Tab. 5 for manufacture/dealer's charge for warranty price with variation of the manufacture estimated product failure intensity over the expected lifetime of the product.

Table 5 Warranty price $(W)$ in $\$$ for different manufacturer's failure intensity

\begin{tabular}{c|cccc}
\hline$\lambda_{m}$ & 0.125 & 0.225 & 0.325 & 0.443 \\
\hline$W$ & 2.65 & 8.53 & 17.68 & 32.17 \\
\hline
\end{tabular}

Analysis Tab. 5 shows that the manufacturer's charge for warranty price increases with the increase of $\lambda_{m}$. This implies that, the higher the manufacturer estimated failure intensity, the higher the manufacturer charge for the warranty price to meet the higher rate of failure and the more warranty claims. Conversely, less failure intensity results in less warranty claims and the manufacturer will be interested to offer less warranty price.

(3) Effect buyer's repair costs on manufacturer set warranty price

Now, the effect of buyer's repair costs on the manufacturer/dealer's charge for warranty price can be analyzed by varying the buyers repair costs for each occasion. Let buyer's repair cost $r_{b}$ be a range of $\$ 40$ to $\$ 80$ per occasion. It is also assumed that the buyer's repair cost remain constant for each case. Let the manufacturer/dealer risk parameters ' $a$ ' is kept constant as 0.4 . All other variables are kept as above. The computer program generates Tab. 6 exhibits the effect of buyer's cost of repair over the manufacture/dealer's charge for warranty price.

Table 6 Buyer's repair cost vs warranty price $(W)$ in $\$$

\begin{tabular}{c|ccccccc}
\hline$r_{b}$ & 40 & 45 & 50 & 55 & 60 & 70 & 80 \\
\hline$W$ & 32.16 & 31.36 & 30.59 & 29.84 & 29.13 & 27.78 & 26.53 \\
\hline
\end{tabular}

An analysis of this table implies that the manufacturer's charge for warranty price decreases with the increase of the of the buyer's cost of repair. This occurs because when the buyer's repair cost is higher (considering buyer's repair cost is same when it is carried by the manufacturer or by the third party repairer), the more buyer will prefer warranty and this in turns provides an opportunity for the manufacturer to charge a lower warranty price to compete effectively in the market. The manufacturer/dealer can make more money from the warranty sale to avoid competition in the repair market.

(4) Effect of buyer rate of return ( $k$ ) to the manufacture/dealer for repair on warranty price

A portion of non-warranted buyer may come back to the original manufacturer/dealer for repair of their failed products. Here, the effect of buyer rate of return $(k)$ to the manufacture/dealer for repair on the warranty price is analyzed. For this purpose, the rate of returning buyers $k$ is varied from $0 \%$ to $100 \%$. This implies different values of $k$ ranging from 0 to 1 . The computer program generates the Tab. 7 which shows the effect of buyer's rate of return over the manufacture's charge for warranty price.

Table 7 Buyer rate of return $(k)$ vs warranty price $(W)$

\begin{tabular}{c|cccccc}
\hline$k$ & 0.01 & 0.2 & 0.4 & 0.6 & 0.8 & 1 \\
\hline$W$ & 34 & 32.165 & 30.36 & 28.659 & 27 & 25 \\
\hline
\end{tabular}

Analysis of Tab. 7 shows that the manufacturer's charge for warranty price decreases with the increase of the rate of buyer's return to the original manufacturer/dealer for repair. This implies that the more the buyer come back to the manufacturer for repair, the more the manufacturer will encourage the buyers to buy the warranty.

\section{Contributions and future works}

Risk attitudes of both buyers and manufacturer/dealers to lifetime warranty policies have been discussed in this paper. Lifetime warranty cost models for product with time dependent failure rate are developed which incorporate risk preferences for optimal warranty price using the manufacturer's utility function for manufacturer's price and the buyer's utility function for repair cost. The proposed models are then analyzed using illustrative numerical examples with respect to buyer's and the manufacturer/dealer's risk preferences. The analysis shows that the more a buyer is risk avert, the higher is his acceptance level of warranty price. On the other hand, the more a manufacturer is risk avert, the more he/she sets warranty price up for the product. Similarly, higher repair costs, and the perception of higher intensity of product failures encourage a buyer to accept higher warranty price. Higher failure intensity also forces a manufacturer to charge higher warranty price but the more return of non-warranted buyers to the original manufacturer for rectification of their failed products may encourage manufacturer to lower it charge for warranty to compete effectively in the market. These models can be useful for manufacturers when making decisions about warranty pricing.

There is a scope for future research with other forms of failure distributions, impact of preventive maintenance and scope for replacements during lifetime with possible tradeins. Another possible extension is to include discounting of the future repair costs and corrections for inflation which are important for products sold with long service life.

\section{References}

[1] Chattopadhyay, G. and Rahman, A. (2007). Development of Lifetime Warranty Policies and Cost Models for Free Replacement Lifetime Warranty (FRLTW) Policy. Reliability Engineers and System Safety. Published On-Line on the 7th march, 2007, Elsevier.

[2] Chun, Y. and Tang, K. (1995). Determining the Optimal Warranty Price Based on the Producer's and Customer's 
Risk Preferences. European Journal of Operational Research, 85:97-110.

[3] Keeney, R. and Raiffa, H. (1976). Decisions with Multiple Objectives: Preferences and Value Tradeoffs. Wiley, New York.

[4] Menezes, M. (1989). The impact of product warranties on consumer preferences. A draft paper submitted to Harvard Business School (Report No. 90-003).

[5] Moskowitz, H. and Plante, R. (1984). Effect of Risk Aversion on Single Sample Attribute Inspection Plans. Management Science, 30:1226-1237.

[6] Rahman, A. and Chattopadhyay, G. (2006). Review of Long Term warranty policies. Asia Pacific Journal of Operational
Research, 22(4):453-473.

[7] Ritchken, P. and Tapiero, C. (1986). Warranty design under buyer and seller risk aversion. Naval Research Logistics Quarterly, 33:657-671.

[8] Wells, C. (1985). Determining the Future Costs of Lifetime Warranty. IIE Transactions, 19(2):178-181.

[9] Zhou, Z., Li, Y., and Tang, K. (2009). Dynamic pricing and warranty policies for products with fixed lifetime. European Journal of Operational Research, 196:940-948.

[10] Zlobec, S. (2004). Jensen's Inequality for Nonconvex Functions. Mathematical Communications, 9:119-124. 
